hep-th/9506104

BONN-TH-95-11

IFP-507-UNC

June 1995

\title{
Exactly Solvable $(0,2)$ Supersymmetric String Vacua With GUT Gauge Groups
}

\author{
Ralph Blumenhagen $^{1}$ and Andreas Wißkirchen ${ }^{2}$ \\ ${ }^{1}$ Institute of Field Physics, Department of Physics and Astronomy, \\ University of North Carolina, Chapel Hill NC 27599-3255, USA \\ ${ }^{2}$ Physikalisches Institut der Universität Bonn, Nußallee 12, 53115 Bonn, Germany
}

\begin{abstract}
We present a construction of modular invariant partition functions for heterotic $(0,2)$ supersymmetric classical string vacua. This generalization of Gepner's construction yields GUT gauge groups $E_{6}, S O(10), S U(5)$ and $S U(3) \times S U(2) \times U(1)^{r}$, respectively. By calculating the massless spectrum of some of these models we find strong indications that they correspond to $(0,2)$ string vacua discussed recently in the context of CYM/LG phases.
\end{abstract}

1 e-mail: blumenha@physics.unc.edu

2 e-mail: wisskirc@avzw01.physik.uni-bonn.de 


\section{Introduction}

Due to the lack of a nonperturbative formulation of string theory we are still restricted to a perturbative search for reasonable string vacua. In the last years classical solutions with $N=1$ space-time supersymmetry have been studied intensively. A necessary condition is that the non flat space-time directions are compactified on a Calabi-Yau manifold (CYM) $[5]$ or that the internal conformal field theory (CFT) has $(0,2)$ world sheet supersymmetry [2], respectively. Besides early indications that generic $(0,2)$ string models might be destabilized by world sheet instantons [6], the symmetric $(2,2)$ models are much easier to handle, so that most effort focused only on their investigation. The implied restriction for the nonlinear $\sigma$ model is that the spin connection is identified with the gauge connection breaking one of the $E_{8}$ factors down to $E_{6}$. However, ever since the work of Witten [19] in 1986 as well as Distler and Greene [7] in 1988, it has been known that weakening the latter identification leads to more realistic GUT gauge groups like $S O(10)$ or $S U(5)$. At the same time CFT models with different kinds of gauge groups have been constructed using free bosons, free fermions or orbifold techniques [1]. In modern terminology those examples with $N=1$ space-time supersymmetry are of type $(0,2)$. For the $(0,2) \mathrm{CY}$ models of Witten, Distler and Greene the left moving fermions of the $\sigma$ model are not any longer sections of the tangent bundle of the CYM, but of a more general stable holomorphic vector bundle of rank four or five, respectively. Since there were those already mentioned reasonable doubts about the consistency at all, these models lost their attraction very fast.

A revival of these models was initiated by Witten's work [20] on the correspondence between nonlinear $\sigma$ models on CYMs and orbifolds of Landau-Ginzburg (LG) models with isolated singularities. Applying his techniques also to the $(0,2)$ case yielded a LG description which allowed one to obtain more detailed information about the properties of a possible conformal fixed point [8]. In [9] it was shown that at least for marginal deformations of $(2,2)$ models by gauge singlets one gets a bona fide CFT. Recently, Silverstein and Witten [18] argued that even for all $(0,2)$ models described by linear $\sigma$ models, the CFTs exist. Nevertheless, the explicit construction of such CFTs was still unclear. Fortunately, some aspects of the structure of these CFTs can already be explored in the LG framework [8]. Due to the left moving R-invariance there exists a left moving $U(1)$ current, the spectral flow operator of which extends $S O(8)$ to $S O(10)$ or $S O(6)$ to $S U(5)$, respectively. Furthermore, the central charges for the left and right moving sector can be calculated correctly; for $S O(10)$ they are $(c, \bar{c})=(10,9)$ and for $S U(5)$ one obtains $(c, \bar{c})=(11,9)$. Thus, it seems to be quite a tough problem to systematically construct modular invariant partition functions for this class of purely heterotic CFTs.

In this paper we present a class of CFTs which satisfies all the conditions mentioned above and which exhibits net numbers of generations which can be reproduced using the CYM/LG framework. One well known way of building new modular invariant partition functions is the simple current technique developed by Schellekens and Yankielowicz $[15,16]$. In the spirit of an idea mentioned in [17] we show that it is also suitable for the construction of the desired $(0,2)$ string vacua. Using light cone gauge, for $S O(10)$ we start with a diagonal invariant partition function of a $(c, \bar{c})=(24,24)$ CFT, which contains the fourdimensional space-time part, an internal $(c, \bar{c})=(9,9) N=2$ supersymmetric part written as a non supersymmetric CFT, a $U(1)_{2}$ part and the Kac-Moody algebra $S O(8) \times E_{8}$ of 
level one. Then we use simple current projections on the right moving side to extend firstly $S O(8) \times U(1)_{2}$ to $S O(10)$. This allows to apply the bosonic string map to yield a right moving superstring with $\bar{c}=12$. The idea of starting only with a subalgebra of $S O(10) \times E_{8}$ and extending it has already been mentioned in [17] but not carried out further. The new feature in our construction is essentially the $U(1)_{2}$ factor. Afterwards, we project onto $N S$ $N S$ and $R$ - $R$ couplings guaranteeing that we choose the 'supersymmetric tensor product' on the r.h.s. The last operation to be carried out on the right is the GSO projection onto even overall $U(1)$ charges. If we would stop at this stage we would get nothing else but the usual Gepner models with $E_{6}$ gauge group [11]. However, because of the new $U(1)_{2}$ factor there occur new possibilities of preventing all the right moving operations to act also on the left. Thus, we divide out the most complicated simple current one can think of containing both pieces of $N S$ sectors and $R$ sectors. In general, this breaks the left moving $N=2$ supersymmetry and the $E_{6}$ gauge group. The last step is to perform the left moving GSO projection extending $S O(8) \times U(1)_{c=9} \times U(1)_{2}$ to $S O(10) \times U(1)$. Note that this extension is different from the one carried out on the r.h.s. Finally, we arrive at a modular invariant partition function with gauge group $S O(10)$ and a $(c, \bar{c})=(10,9)$ CFT in the internal sector. Since there occur new combinations of left moving excitations which are massless, in general the spectrum of the string changes drastically. Comparing these spectra to those of the Distler/Kachru models we found indications that we have really constructed CFTs, describing certain points in the moduli space of the latter models. The $S U(5)$ case is quite analogous, instead of one $U(1)_{2}$ CFT one uses two such factors. As expected for consistent string vacua, the gauge anomaly cancellation comes out automatically [14]. Of course, the above series can be extended further, three $U(1)_{2}$ factors generically yield the non GUT gauge group $S U(3) \times S U(2)$. In general, in four dimensions our construction allows all $E_{r}$ gauge groups with $3 \leq r \leq 6$, which are defined by removing successively one simple root of a long leg of the $E_{6}$ Dynkin diagram. In six and eight space-time dimensions the usual Gepner construction yields the remaining exceptional gauge groups $E_{7}$ and $E_{8}$, respectively.

This paper is organized as follows. In section 2 we review some basic facts about the simple current technique. Then we present our construction of $(0,2)$ modular invariant partition functions. The $S O(10)$ case is discussed in detail, whereas $S U(5)$ and $S U(3) \times S U(2)$ are dealt with rather briefly. A discussion of the general massless spectra follows in section 6 . In section 7 we present the results of a computer calculation for some exemplary models like the quintic and compare them to the results gained by CYM/LG techniques.

\section{Review of the simple current technique}

This chapter contains only a very short review of the work of Schellekens and Yankielowicz about generating new modular invariant partitions using simple currents. For a more detailed discussion we refer the reader to the original literature $[15,16,17]$. Suppose there is given a rational conformal field theory (RCFT) with at least one modular invariant partition function, e.g. the diagonal one. If this RCFT contains a simple current $J$, i.e. $J \times \Phi_{i}=\Phi_{j}$ for every highest weight representation $\Phi_{i}$ of the chiral algebra in the model, then one can obtain a new modular invariant in the following way: First, define the index $N$ of the simple current $J$ to be the smallest integer so that $J^{N}=\mathbb{1}$. Furthermore, the 
monodromy parameter $r$ is determined by the conformal dimension of $J$ :

$$
h(J)=\frac{r(N-1)}{2 N} \bmod 1
$$

so that $r$ is defined modulo $N$ for $N$ odd and modulo $2 N$ for $N$ even. Next, one defines the (monodromy) charge of a primary field $\Phi$ :

$$
Q(\Phi)=h(\Phi)+h(J)-h(J \times \Phi) \bmod 1
$$

which takes values $\frac{t}{N}, t \in \mathbb{Z}$. By the action of the simple current all primaries of the RCFT are arranged in orbits $\Phi, J \times \Phi, \ldots, J^{d} \times \Phi$, where $d$ is a divisor of $N$. The charges of the fields occurring in an orbit are $\frac{t+r n}{N} \bmod 1$.

If one can choose $r$ to be even, one can form a new modular invariant partition function

$$
Z(\tau, \bar{\tau})=\sum_{k, l} \chi_{k}(\tau) M_{k l} \chi_{l}(\bar{\tau})
$$

with the matrix $M$ determined by the orbits and the charges of the fields with respect to the simple current $J$ :

$$
M_{k l}=\sum_{p=1}^{N} \delta\left(\Phi_{k}, J^{p} \Phi_{l}\right) \delta^{1}\left(\hat{Q}\left(\Phi_{k}\right)+\hat{Q}\left(\Phi_{l}\right)\right)
$$

where $\delta^{1}(x)=1$ for $x \in \mathbb{Z}$ and zero otherwise. The slightly modified charge $\hat{Q}$ is defined on each orbit by

$$
\hat{Q}\left(J^{n} \Phi\right)=\frac{t+r n}{2 N} \bmod 1 .
$$

Two different kinds of invariants occur. On the one hand, those that result from simple currents of integer conformal dimension. These can be regarded as diagonal invariants for a subset of orbits having integer monodromy charge. Thus, some of the original representations are really projected out. On the other hand, simple currents of non integer dimension lead to invariants corresponding to automorphisms of the fusion algebra which in particular means that only the pairing of the left and right moving sector changes. Obviously, the product of two matrices (2.4) also defines a modular invariant partition function which can be divided consistently by an integer in order to guarantee the vacuum to appear only once. Thus, in general one is allowed to form partition functions like

$$
Z(\tau, \bar{\tau}) \sim \vec{\chi}(\tau) M\left(J_{n}\right) \ldots M\left(J_{2}\right) M\left(J_{1}\right) \vec{\chi}(\bar{\tau})
$$

The method of simple currents provides one with a powerful laboratory for the construction of modular invariant partition functions. In [17] it has extensively been used for the construction of four-dimensional, $N=1$ space-time supersymmetric string vacua with an internal $(c, \bar{c})=(9,9) \mathrm{CFT}$. There, it already appeared that in general one gets only $(0,2)$ 
world sheet supersymmetry. However, unlike to our models the gauge group $S O(10)$ is linearly realized and not a result of a left moving GSO projection implying the internal left moving central charge to be also $c=9$ and not $c=10$. In the following sections we investigate whether this large laboratory can also provide us with models of the Distler/Kachru type [8].

\section{String models with $(0,2)$ supersymmetry and gauge group $\mathrm{SO}(10)$}

In this section we make use of the simple current technique to find modular invariant partition functions which satisfy all the properties known for the conformal fixed points of the $(0,2)$ string vacua. First, we concentrate on the $S O(10)$ case resulting from choosing a stable vector bundle of rank four. In [8] the following information about the CFT has been extracted from an LG analysis:

(a) The left and right conformal anomalies of the internal CFT are $(c, \bar{c})=(10,9)$.

(b) Besides the right moving $U(1)$ current which is part of the right moving $N=2$ Virasoro algebra there exists a left moving $U(1)$ current satisfying the following operator product expansion (OPE):

$$
J(z) J(w)=\frac{4}{(z-w)^{2}}+\text { reg. }
$$

(c) Only the subset $S O(8) \times U(1) \subset S O(10)$ of the gauge group is linearly realized, the remaining roots are generated by taking orbits with respect to the spectral flow of conformal dimension $(h, q)=\left(\frac{1}{2}, 2\right)$.

Furthermore, we know that there is still a CYM in the model. As we have learned from Gepner's work on $(2,2)$ models [11], some of them correspond to tensor products of unitary $N=2$ models. Using light cone gauge we start with the diagonal partition function for the $(c, \bar{c})=(24,24)$ CFT model shown in Table 3.1.

\begin{tabular}{|l|l|l|}
\hline part & $c$ & $\bar{c}$ \\
\hline $4 D$ space-time, $X^{\mu}$ & 2 & 2 \\
\hline$N=2$ Virasoro & 9 & 9 \\
\hline$U(1)_{2}$ & 1 & 1 \\
\hline gauge group $S O(8) \times E_{8}$ & 12 & 12 \\
\hline
\end{tabular}

Table $3.1 \quad$ Underlying CFT for $S O(10)$

The remarkable change compared to Gepner's models is the appearance of a free boson compactified on a circle of radius $R=2$ denoted as $U(1)_{2}$. The diagonal partition function for this part can easily be expressed in terms of $\Theta$-functions:

$$
Z_{U(1)_{2}}(\tau, \bar{\tau})=\sum_{m=-1}^{2} \Theta_{m, 2}(\tau) \Theta_{m, 2}(\bar{\tau}) .
$$


Note, that this is nothing else but the partition function of a Dirac fermion. The fusion rules are quite simple:

$$
\left[\Phi_{m, 2}\right] \times\left[\Phi_{n, 2}\right]=\left[\Phi_{m+n, 2}\right] \bmod 4 .
$$

The current is $j_{U(1)_{2}}=i \partial \phi$ and satisfies the following OPE:

$$
j_{U(1)_{2}}(z) j_{U(1)_{2}}(w)=\frac{1}{(z-w)^{2}}+\text { reg. }
$$

Furthermore, even though $U(1)_{2}$ is surely not $N=2$ supersymmetric, there exists a spectral flow between the sector of even index $m$ and odd index $m$ or between the $N S$ sector and the $R$ sector of the Dirac fermion, respectively. The spectral flow operator is $\exp \left(\frac{i \phi(z)}{2}\right)$ and has conformal dimension and charge $(h, q)=\left(\frac{1}{8}, \frac{1}{2}\right)$. Now, it becomes obvious why we have chosen this special $c=1$ theory. Combining it with the left moving $c=9$ theory offers the possibility to define an overall $U(1)$ current $J$ which satisfies the conditions $(b)$ and $(c)$. The sum of the $N=2$ current $j_{c=9}=i \sqrt{3} \partial \Phi(z)$ and the $U(1)_{2}$ current satisfies the OPE in $(b)$ and the left moving spectral flow operator is given by

$$
\Sigma_{c=10}(z)=e^{i \frac{\sqrt{3}}{2} \Phi(z)} \otimes e^{i \frac{1}{2} \phi(z)}
$$

Later on we will see that taking orbits with respect to this spectral flow operator really extends $S O(8) \times U(1)$ to $S O(10)$.

Now we proceed by discussing the right moving sector. To this end let us recall some facts about the representations of $S O(2 n)$ Kac-Moody algebras at level $k=1$. All we need for the following discussion is summarized in Table 3.2.

\begin{tabular}{|l|l|l|l|}
\hline character & $h$ & $q \bmod 2$ & degeneracy \\
\hline$\chi_{0}=\frac{1}{2}\left(\left(\frac{\vartheta_{3}}{\eta}\right)^{n}+\left(\frac{\vartheta_{4}}{\eta}\right)^{n}\right)$ & 0 & 0 & 0 \\
$\chi_{v}=\frac{1}{2}\left(\left(\frac{\vartheta_{3}}{\eta}\right)^{n}-\left(\frac{\vartheta_{4}}{\eta}\right)^{n}\right)$ & $\frac{1}{2}$ & 1 & $2 n$ \\
$\chi_{s}=\frac{1}{2}\left(\left(\frac{\vartheta_{2}}{\eta}\right)^{n}+\left(\frac{\vartheta_{1}}{\eta}\right)^{n}\right)$ & $\frac{n}{8}$ & $\frac{n}{2}$ & $2^{n-1}$ \\
$\chi_{c}=\frac{1}{2}\left(\left(\frac{\vartheta_{2}}{\eta}\right)^{n}-\left(\frac{\vartheta_{1}}{\eta}\right)^{n}\right)$ & $\frac{n}{8}$ & $\frac{n}{2}-1$ & $2^{n-1}$ \\
\hline
\end{tabular}

Table 3.2 Representations of $S O(2 n)_{1}$

The charge $q$ is taken with respect to the sum of all Cartan elements of the Lie algebra $S O(2 n)$ and $\vartheta_{i}$ denotes the Jacobi $\vartheta$-functions. The fusion rules for the representations are different for $n$ even and $n$ odd as one can read off from Table 3.3.

\begin{tabular}{|c|cccc|c|cccc|}
\hline$n$ odd & 0 & $v$ & $s$ & $c$ \\
\hline 0 & 0 & $v$ & $s$ & $c$ \\
$v$ & $v$ & 0 & $c$ & $s$ \\
$s$ & $s$ & $c$ & $v$ & 0 \\
$c$ & $c$ & $s$ & 0 & $v$ \\
\hline
\end{tabular}

Table $3.3 \quad$ Fusion rules for $S O(2 n)_{1}$ 
In order to apply the bosonic string map for $S O(10) \times E_{8} \rightarrow S O(2)$

$$
\begin{array}{ll}
\chi_{0}^{S O(10) \times E_{8}} \rightarrow \chi_{v}^{S O(2)}, & \chi_{v}^{S O(10) \times E_{8}} \rightarrow \chi_{0}^{S O(2)} \\
\chi_{s}^{S O(10) \times E_{8}} \rightarrow-\chi_{c}^{S O(2)}, & \chi_{c}^{S O(10) \times E_{8}} \rightarrow-\chi_{s}^{S O(2)}
\end{array}
$$

on the r.h.s. we have to extend $S O(8) \times U(1)_{2}$ to $S O(10)$. This can be done by using the simple current

$$
J_{(1 \times 8 \rightarrow 10)}=\Phi_{2,2}^{U(1)_{2}} \otimes \Phi_{v}^{S O(8)},
$$

which generates the following orbits:

$$
\begin{aligned}
& \chi_{0}^{S O(10)}=\chi_{0}^{S O(8)} \Theta_{0,2}+\chi_{v}^{S O(8)} \Theta_{2,2} \\
& \chi_{v}^{S O(10)}=\chi_{0}^{S O(8)} \Theta_{2,2}+\chi_{v}^{S O(8)} \Theta_{0,2} \\
& \chi_{s}^{S O(10)}=\chi_{s}^{S O(8)} \Theta_{1,2}+\chi_{c}^{S O(8)} \Theta_{-1,2} \\
& \chi_{c}^{S O(10)}=\chi_{c}^{S O(8)} \Theta_{1,2}+\chi_{s}^{S O(8)} \Theta_{-1,2} .
\end{aligned}
$$

Now we can proceed on the right moving side in the same way as in Gepner's construction. For the $c=9$ part we choose tensor products of unitary representations of the $N=2$ super Virasoro algebra,

$$
\begin{aligned}
& c=\frac{3 k}{k+2}, \quad h_{m, s}^{l}=\frac{l(l+2)-m^{2}}{4(k+2)}+\frac{s^{2}}{8}, \quad q_{m, s}^{l}=-\frac{m}{k+2}+\frac{s}{2}, \\
& k \in \mathbb{N}, \quad 0 \leq l \leq k, \quad-1 \leq s \leq 2, \quad-l+\epsilon \leq m \leq l+\epsilon, \quad l+m+s=0 \bmod 2
\end{aligned}
$$

with $\epsilon=0$ for $s \in\{0,2\}$ ( $N S$ sector) and $\epsilon=1$ for $s \in\{-1,1\}$ ( $R$ sector), respectively. Here we have split the characters in the usual way into two non supersymmetric pieces:

$$
\chi_{m}^{l}=\chi_{m, s}^{l}+\chi_{m, s+2}^{l} .
$$

Then, in order to ensure that we are actually dealing with an $N=2$ supersymmetric model, we have to impose further projections

$$
J_{i}=G_{i} \otimes \Phi_{v}^{S O(8)}
$$

where $G_{i}$ means the supercurrent in the $i$-th factor of the tensor product. These projections allow only couplings between same kinds of sectors. The last step to be carried out on the r.h.s. is the right moving GSO projection onto states with even overall charge. The necessary simple current is

$$
J_{G S O_{R}}=\Sigma_{c=9} \otimes \Phi_{1,2}^{U(1)_{2}} \otimes \Phi_{s}^{S O(8)} .
$$

where $\Sigma_{c=9}$ denotes the spectral flow operator of dimension $(h, q)=\left(\frac{3}{8}, \frac{3}{2}\right)$ of the internal $c=9 \mathrm{CFT}$. In a concrete model $\Sigma_{c=9}$ simply contains one $\Phi_{1,1}^{0}$ primary field for each factor. So far, the partition function looks like

$$
Z \sim \vec{\chi}(\tau) M\left(J_{G S O_{R}}\right) \prod_{i} M\left(J_{i}\right) M\left(J_{(1 \times 8 \rightarrow 10)}\right) \vec{\chi}(\bar{\tau})
$$


which produces exactly the ordinary Gepner models, for all projections act also on the left. Thus, in order to get something new we have to prevent this by introducing more simple currents from the left which do not commute with the simple currents in (3.13). Which simple currents are suitable depends on the concrete model one is dealing with. However, on account of the new $U(1)_{2}$ factor there occur simple currents which are not present in the Gepner case. In general we are interested in simple currents which both break the left moving $N=2$ supersymmetry and the $E_{6}$ gauge group resulting from the $J_{G S O_{R}}$ projection. Suppose now we have found such fields $\Upsilon_{l}$. What remains is only the left moving GSO projection which is performed by the simple current

$$
J_{G S O_{L}}=\Sigma_{c=10} \otimes \Phi_{s}^{S O(8)}
$$

which is actually the same as for the right moving GSO projection. However, since the simple currents $J_{i}$ and $J_{(1 \times 8 \rightarrow 10)}$ do not any longer act on the left, it does not yield an extension of the gauge group to $E_{6}$ but only to $S O(10)$. On the level of characters this can be seen by using a general theorem about orbits of spectral flows of chiral dimension $(h, q)=\left(\frac{k}{2}, k\right)[13]$. Since in the $N S$ sector all orbits contain only states with integral charge, Hermite's lemma $†$ tells us that every orbit can be expanded into a finite number of $z$ dependent functions

$$
f_{Q, k}(q, z)=\frac{1}{\eta(q)} \sum_{n \in \mathbb{Z}} q^{\frac{k}{2}\left(n+\frac{Q}{k}\right)^{2}} z^{k\left(n+\frac{Q}{k}\right)}, \quad Q \bmod k
$$

where the coefficients depend only on the variable $q$. In our case the chiral spectral flow is twice the flow $\Sigma_{c=10}$ and therefore has dimension $(h, q)=(2,4)$. Consequently, there are only four invariant functions which can also be written in terms of $\Theta$ functions:

$$
f_{i, 4}(q, z)=\frac{1}{\eta(q)}\left(\Theta_{2 i, 8}(q, z)+\Theta_{2(i+4), 8}(q, z)\right), \quad-1 \leq i \leq 2
$$

Note, that $f_{0,4}, f_{2,4}$ have even charge and $f_{-1,4}, f_{1,4}$ odd charge. Since $\Sigma_{c=10}$ acts on the invariant functions by

$$
\Sigma_{c=10}: f_{i, 4} \rightarrow f_{i+2,4}
$$

every orbit under $J_{G S O_{L}}$ with even charge can be expanded in the following way:

$$
\begin{aligned}
\chi_{\text {orb }}^{j}= & \chi_{0}^{S O(8)}\left[f_{0,4} A_{0}^{j}+f_{2,4} A_{2}^{j}\right]+\chi_{v}^{S O(8)}\left[f_{1,4} A_{1}^{j}+f_{-1,4} A_{-1}^{j}\right] \\
& \chi_{s}^{S O(8)}\left[f_{2,4} A_{0}^{j}+f_{0,4} A_{2}^{j}\right]+\chi_{c}^{S O(8)}\left[f_{-1,4} A_{1}^{j}+f_{1,4} A_{-1}^{j}\right] .
\end{aligned}
$$

$\dagger$ For $a \in \mathbb{N}$ and $0 \leq b \leq a$ and $\delta= \pm 1$ be fixed: If $f(z)=f(z, q)$ is a Laurent series in $z$ and satisfies $f(z q, q)=\frac{\bar{\delta}}{z^{a} q^{\frac{b}{2}}} f(z, q)$, then $\{f(z)\}$ is an $a$-dimensional vector space and one can choose the following basis: $z^{\rho} \sum_{n \in \mathbb{Z}} \delta^{n} z^{a n} q^{\frac{a}{2} n^{2}+\left(\rho+\frac{(b-a)}{2}\right) n}$ with $\rho=0,1, \ldots, a-1$. 
Reordering yields

$$
\begin{aligned}
\chi_{\text {orb }}^{j}= & {\left[\chi_{0}^{S O(8)} f_{0,4}+\chi_{s}^{S O(8)} f_{2,4}\right] A_{0}^{j}+\left[\chi_{0}^{S O(8)} f_{2,4}+\chi_{s}^{S O(8)} f_{0,4}\right] A_{2}^{j}+} \\
& {\left[\chi_{v}^{S O(8)} f_{1,4}+\chi_{c}^{S O(8)} f_{-1,4}\right] A_{1}^{j}+\left[\chi_{v}^{S O(8)} f_{-1,4}+\chi_{c}^{S O(8)} f_{1,4}\right] A_{-1}^{j} . }
\end{aligned}
$$

After some algebra neglecting the $z$ dependence this can be written as

$$
\chi_{\text {orb }}^{j}=\chi_{0}^{S O(10)} A_{0}^{j}+\chi_{v}^{S O(10)} A_{2}^{j}+\chi_{s}^{S O(10)} A_{1}^{j}+\chi_{c}^{S O(10)} A_{-1}^{j}
$$

showing explicitly the extension of the gauge group to $S O(10)$. Summarizing, the entire model has the form

$$
Z \sim \vec{\chi}(\tau) M\left(J_{G S O_{L}}\right) \prod_{l} M\left(\Upsilon_{l}\right) M\left(J_{G S O_{R}}\right) \prod_{i} M\left(J_{i}\right) M\left(J_{(1 \times 8 \rightarrow 10)}\right) \vec{\chi}(\bar{\tau})
$$

and by construction exhibits all the properties required at the beginning of this section. The remaining question is whether one can really find simple currents $\Upsilon_{l}$ which break both the left moving supersymmetry and the $E_{6}$ gauge group. An explicit computer calculation shows that generically this is not difficult. Apparently, at least for the moment we have no other criteria to decide what the influence of a set of simple currents $\Upsilon_{l}$ is than to perform the explicit calculation. Of course, those which act trivially on the $U(1)_{2}$ should correspond to ordinary orbifold constructions of the CYM, whereas others reflect the choice of a different vector bundle for the left moving $\sigma$ model fermions and thus reducing the rank of the gauge group. In section 7 we present some first results of an explicit calculation showing what kinds of spectra one can expect from the models in (3.21).

\section{String models with $(0,2)$ supersymmetry and gauge group $\mathrm{SU}(5)$}

The generalization of the above construction to $S U(5)$ is straightforward, so that it will be presented more briefly. The CYM/LG analysis reveals the following information about the conformal fixed point:

(a) The left and right conformal anomalies of the internal CFT are $(c, \bar{c})=(11,9)$.

(b) The OPE of the left moving $U(1)$ current is

$$
J(z) J(w)=\frac{5}{(z-w)^{2}}+\text { reg. }
$$

(c) The subset $S O(6) \times U(1) \subset S U(5)$ is linearly realized, orbits with respect to the spectral flow of conformal dimension $(h, q)=\left(\frac{5}{8}, \frac{5}{2}\right)$ generate the missing roots.

Analogously to the former case, we suggest the ansatz for a relevant model presented in Table 4.1. 


\begin{tabular}{|l|l|l|}
\hline part & $c$ & $\bar{c}$ \\
\hline \hline $4 D$ space-time, $X^{\mu}$ & 2 & 2 \\
\hline$N=2$ Virasoro & 9 & 9 \\
\hline$U(1)_{2} \otimes U(1)_{2}$ & 2 & 2 \\
\hline gauge group $S O(6) \times E_{8}$ & 11 & 11 \\
\hline
\end{tabular}

Table $4.1 \quad$ Underlying CFT for $S U(5)$

On the r.h.s the extension of $U(1)_{2} \times U(1)_{2} \times S O(6)$ to $S O(10)$ can be achieved by the following two simple currents:

$$
\begin{aligned}
& J_{1 \times 1 \times 6 \rightarrow 10}^{1}=\Phi_{2,2}^{U(1)_{2}} \otimes \Phi_{0,2}^{U(1)_{2}} \otimes \Phi_{v}^{S O(6)} \\
& J_{1 \times 1 \times 6 \rightarrow 10}^{2}=\Phi_{0,2}^{U(1)_{2}} \otimes \Phi_{2,2}^{U(1)_{2}} \otimes \Phi_{v}^{S O(6)}
\end{aligned}
$$

The projections ensuring $N=2$ supersymmetry on the right moving side are still given by

$$
J_{i}=G_{i} \otimes \Phi_{v}^{S O(6)}
$$

and the GSO projection leading to $N=1$ space-time supersymmetry is

$$
J_{G S O_{R}}=\Sigma_{c=9} \otimes \Phi_{1,2}^{U(1)_{2}} \otimes \Phi_{1,2}^{U(1)_{2}} \otimes \Phi_{s}^{S O(6)} .
$$

So far, the model looks like

$$
Z \sim \vec{\chi}(\tau) M\left(J_{G S O_{R}}\right) \prod_{i} M\left(J_{i}\right) \prod_{j=1}^{2} M\left(J_{(1 \times 1 \times 6 \rightarrow 10)}^{j}\right) \vec{\chi}(\bar{\tau})
$$

The left moving $U(1)$ current is the sum of the $N=2$ current and the two $U(1)_{2}$ currents and in particular satisfies the OPE (4.1). The associated spectral flow operator of dimension $(h, q)=\left(\frac{5}{8}, \frac{5}{2}\right)$ is

$$
\Sigma_{c=11}(z)=e^{i \frac{\sqrt{3}}{2} \Phi(z)} \otimes e^{i \frac{1}{2} \phi_{1}(z)} \otimes e^{i \frac{1}{2} \phi_{2}(z)}
$$

In order to perform the left moving GSO projection we use the simple current

$$
J_{G S O_{L}}=\Sigma_{c=9} \otimes \Phi_{1,2}^{U(1)_{2}} \otimes \Phi_{1,2}^{U(1)_{2}} \otimes \Phi_{s}^{S O(6)}
$$

again. In the $N S$ sector there exist five series invariant with respect to the square of the flow $\Sigma_{c=11}$

$$
f_{Q, 5}(q, z)=\frac{1}{\eta(q)} \sum_{m \in \mathbb{Z}} q^{\frac{5}{2}\left(m+\frac{Q}{5}\right)^{2}} z^{5\left(m+\frac{Q}{5}\right)}, \quad-2 \leq Q \leq 2 .
$$


Similar to the Gepner case [3,10] they are not of definite charge parity, so that we have to use the decomposition into $\Theta$ functions

$$
f_{Q, 5}(q, z)=\frac{1}{\eta(q)}\left(\Theta_{2 Q, 10}(q, z)+\Theta_{2 Q+10,10}(q, z)\right) \quad \bmod 20 .
$$

Taking into account the action of $\Sigma_{c=11}$ on the $\Theta$ functions:

$$
\Sigma_{c=11}: \Theta_{i, 10} \rightarrow \Theta_{i+5,10} \bmod 20
$$

every orbit of even overall charge can be expanded in the following way:

$$
\begin{aligned}
\chi_{\text {orb }}^{j}= & \chi_{0}^{S O(6)}\left[\Theta_{0,10} A_{0}^{j}+\Theta_{-8,10} A_{1}^{j}+\Theta_{8,10} A_{-1}^{j}+\Theta_{4,10} A_{2}^{j}+\Theta_{-4,10} A_{-2}^{j}\right]+ \\
& \chi_{v}^{S O(6)}\left[\Theta_{10,10} A_{0}^{j}+\Theta_{2,10} A_{1}^{j}+\Theta_{-2,10} A_{-1}^{j}+\Theta_{-6,10} A_{2}^{j}+\Theta_{6,10} A_{-2}^{j}\right]+ \\
& \chi_{s}^{S O(6)}\left[\Theta_{5,10} A_{0}^{j}+\Theta_{-3,10} A_{1}^{j}+\Theta_{-7,10} A_{-1}^{j}+\Theta_{9,10} A_{2}^{j}+\Theta_{1,10} A_{-2}^{j}\right]+ \\
& \chi_{c}^{S O(6)}\left[\Theta_{-5,10} A_{0}^{j}+\Theta_{7,10} A_{1}^{j}+\Theta_{3,10} A_{-1}^{j}+\Theta_{-1,10} A_{2}^{j}+\Theta_{-9,10} A_{-2}^{j}\right] .
\end{aligned}
$$

Reordering and the fact that the characters of $S U(5)$ can be written as sums over products of those of $S U(4)$ and $\Theta$ functions at level ten yields

$$
\chi_{\text {orb }}^{j}=\chi_{0}^{S U(5)} A_{0}^{j}+\chi_{10}^{S U(5)} A_{+1}^{j}+\chi_{\overline{5}}^{S U(5)} A_{+2}^{j}+\chi_{10}^{S U(5)} A_{-1}^{j}+\chi_{5}^{S U(5)} A_{-2}^{j} .
$$

This shows very nicely the extension of the gauge group to $S U(5)$. Unlike $E_{6}$ and $S O(10)$ there exist two different representations for chiral space-time fermions, the $\mathbf{1 0}$ and the $\overline{\mathbf{5}}$ which together contains one generation of the standard model. As we will see in the following sections the number of total generations in $\mathbf{1 0}$ and $\overline{\mathbf{5}}$ in general are not the same, whereas the number of net generations are. Thus, the whole partition function is

$$
Z \sim \vec{\chi}(\tau) M\left(J_{G S O_{L}}\right) \prod_{l} M\left(\Upsilon_{l}\right) M\left(J_{G S O_{R}}\right) \prod_{i} M\left(J_{i}\right) \prod_{j=1}^{2} M\left(J_{(1 \times 1 \times 6 \rightarrow 10)}^{j}\right) \vec{\chi}(\bar{\tau})
$$

Apparently, the whole construction can be extended further starting with three copies of $U(1)_{2}$ and the group $S O(4) \times E_{8}$ which extends to $S U(3) \times S U(2) \times E_{8}$. Since at our exactly solvable points there are a lot $U(1)$ factors around, one might get the supersymmetric standard model. However, analogously to the $(2,2)$ case these $U(1)$ factors are believed to exist only at this special point of the moduli space of the CYM and would be broken by a generic marginal deformation. Thus, very sensitive fine tuning is necessary to choose such a special string vacuum. 
The next kind of models are those which exhibit gauge group $E_{3}=S U(3) \times S U(2)$. At certain points of the moduli space $E_{3}$ may be extended by at least some $U(1)$ factors, so that the gauge group contains the standard model. The ansatz is shown in Table 5.1.

\begin{tabular}{|l|l|l|}
\hline part & $c$ & $\bar{c}$ \\
\hline \hline $4 D$ space-time, $X^{\mu}$ & 2 & 2 \\
\hline$N=2$ Virasoro & 9 & 9 \\
\hline$U(1)_{2} \otimes U(1)_{2} \otimes U(1)_{2}$ & 3 & 3 \\
\hline gauge group $S O(4) \times E_{8}$ & 10 & 10 \\
\hline
\end{tabular}

Table 5.1 Underlying CFT for $S U(3) \times S U(2)$

On the r.h.s the extension of $U(1)_{2} \times U(1)_{2} \times U(1)_{2} \times S O(4)$ to $S O(10)$ can be achieved by the following three simple currents:

$$
\begin{aligned}
& J_{1^{3} \times 4 \rightarrow 10}^{1}=\Phi_{2,2}^{U(1)_{2}} \otimes \Phi_{0,2}^{U(1)_{2}} \otimes \Phi_{0,2}^{U(1)_{2}} \otimes \Phi_{v}^{S O(4)} \\
& J_{1^{3} \times 4 \rightarrow 10}^{2}=\Phi_{0,2}^{U(1)_{2}} \otimes \Phi_{2,2}^{U(1)_{2}} \otimes \Phi_{0,2}^{U(1)_{2}} \otimes \Phi_{v}^{S O(4)} \\
& J_{1^{3} \times 4 \rightarrow 10}^{3}=\Phi_{0,2}^{U(1)_{2}} \otimes \Phi_{0,2}^{U(1)_{2}} \otimes \Phi_{2,2}^{U(1)_{2}} \otimes \Phi_{v}^{S O(4)} .
\end{aligned}
$$

The GSO projection is

$$
J_{G S O_{R}}=\Sigma_{c=9} \otimes \Phi_{1,2}^{U(1)_{2}} \otimes \Phi_{1,2}^{U(1)_{2}} \otimes \Phi_{1,2}^{U(1)_{2}} \otimes \Phi_{s}^{S O(4)} .
$$

This simple current is also used to perform the left moving GSO projection. In the NS sector there exist six series invariant with respect to the square of the flow $\Sigma_{c=12}$ :

$$
f_{Q, 6}(q, z)=\frac{1}{\eta(q)} \sum_{m \in \mathbb{Z}} q^{3\left(m+\frac{Q}{6}\right)^{2}} z^{6\left(m+\frac{Q}{6}\right)}, \quad-2 \leq Q \leq 3 .
$$

Thus, every orbit of the left moving GSO projection can be expanded in the following way:

$$
\begin{aligned}
\chi_{\text {orb }}^{j}= & \chi_{0}^{S O(4)}\left[f_{0,6} A_{0}^{j}+f_{2,6} A_{2}^{j}+f_{-2,6} A_{-2}^{j}\right]+ \\
& \chi_{v}^{S O(4)}\left[f_{3,6} A_{3}^{j}+f_{1,6} A_{1}^{j}+f_{-1,6} A_{-1}^{j}\right]+ \\
& \chi_{s}^{S O(4)}\left[f_{3,6} A_{0}^{j}+f_{-1,6} A_{2}^{j}+f_{1,6} A_{-2}^{j}\right]+ \\
& \chi_{c}^{S O(4)}\left[f_{0,6} A_{3}^{j}+f_{-2,6} A_{1}^{j}+f_{2,6} A_{-1}^{j}\right]
\end{aligned}
$$

which can be rewritten in terms of $E_{3}$ characters

$$
\chi_{o r b}^{j}=\chi_{(0,0)}^{E_{3}} A_{0}^{j}+\chi_{(0,2)}^{E_{3}} A_{3}^{j}+\chi_{(3,0)}^{E_{3}} A_{-2}^{j}+\chi_{(\overline{3}, 0)}^{E_{3}} A_{2}^{j}+\chi_{(3,2)}^{E_{3}} A_{-1}^{j}+\chi_{(\overline{3}, 2)}^{E_{3}} A_{1}^{j} .
$$


Thus, there appear four chiral representations. The whole partition function is

$$
Z \sim \vec{\chi}(\tau) M\left(J_{G S O_{L}}\right) \prod_{l} M\left(\Upsilon_{l}\right) M\left(J_{G S O_{R}}\right) \prod_{i} M\left(J_{i}\right) \prod_{j=1}^{3} M\left(J_{1^{3} \times 4 \rightarrow 10}^{j}\right) \vec{\chi}(\bar{\tau})
$$

Remarkably, the above generalized Gepner type construction yields all gauge groups $E_{6}$, $E_{5}=S O(10), E_{4}=S U(5)$ and $E_{3}=S U(3) \times S U(2)$. The extension of the gauge group $S O(2 n) \times U(1)$ to $E_{n+1}$ is schematically represented by the extension of the Dynkin diagrams shown in Figure 5.1.

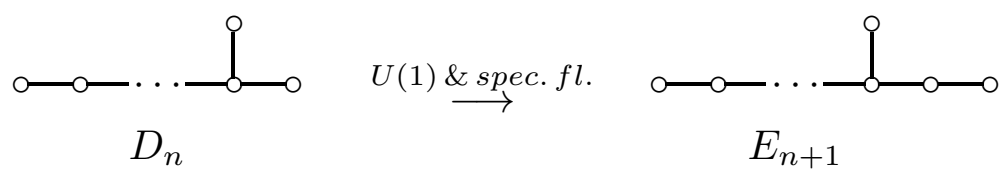

Figure 5.1 Extension of Dynkin diagrams

In order to extract more concrete information about these models we have to calculate some physical quantities. As a first step we concentrate on the massless spectrum, especially the number of generations which can also be calculated in the CYM/LG scheme.

\section{The massless spectrum}

To begin with, there are the universal massless particles of the heterotic string like the graviton, the gravitino and the gluons and gluinos of the gauge group. The coupling of the vacuum on the l.h.s. to the space-time SUSY supercharges

$$
\left(\bar{h}=\frac{3}{8}, \bar{q}\right)_{\bar{c}=9} \otimes\left(\Phi_{1,2}^{U(1)_{2}}\right)^{5-n} \otimes \Phi_{s}^{S O(2 n)}
$$

on the r.h.s. determines the degree of supersymmetry. If there occur $k$ such states one actually deals with $N=k$ space-time supersymmetry. In most examples discussed in section 7 we only have $N=1$ space-time supersymmetry. However, a compactification on $K_{3} \times T^{2}$ yields also $N=2$ supersymmetry which recently has received attention because of duality relations to type II Calabi-Yau compactifications [12].

In addition, for $S O(10)$ the spectrum contains spin zero and spin one particles in the singlet, vector and spinor representations and their corresponding superpartners of spin one half. For the bosons their quantum numbers in the internal $(c, \bar{c})=(10,9)$ CFT are listed in the form $(h, q ; \bar{h}, \bar{q})$ in Table 6.1.

\begin{tabular}{|l|l|l|l|l|}
\hline & $\mathbf{0}$ & $\mathbf{1 0}$ & $\mathbf{1 6}$ & $\overline{\mathbf{1 6}}$ \\
\hline spin 0 & $\left(1,0 ; \frac{1}{2}, \pm 1\right)$ & $\left(\frac{1}{2}, 0 ; \frac{1}{2}, \pm 1\right)$ & $\left(\frac{1}{2}, 1 ; \frac{1}{2}, \pm 1\right)$ & $\left(\frac{1}{2},-1 ; \frac{1}{2}, \pm 1\right)$ \\
\hline spin 1 & $(1,0 ; 0,0)$ & $\left(\frac{1}{2}, 0 ; 0,0\right)$ & $\left(\frac{1}{2}, 1 ; 0,0\right)$ & $\left(\frac{1}{2},-1 ; 0,0\right)$ \\
\hline
\end{tabular}

Table 6.1 Massless spectrum for $S O(10)$ 
Since the right moving sector is the same as in ordinary $(2,2)$ models, the same combinations of primaries from the different factor models contribute to the right moving part of the massless states. Thus, in a concrete calculation one starts with these combinations on the r.h.s. of the chain in (3.21) and follows their way through all the simple currents to determine to which states they couple on the l.h.s. The appearance of spin one particles in the spinor representations indicates an extension of the gauge group, in the $S O(10)$ case usually to $E_{6}$. However, in general this does not mean that also the $N=2$ supersymmetry is restored in the left moving sector.

In the case of $S U(5)$ only the singlet and the four spinor representations occur. The quantum numbers in the internal $(c, \bar{c})=(11,9)$ CFT are listed in Table 6.2.

\begin{tabular}{|l|l|l|l|l|l|}
\hline & $\mathbf{0}$ & $\mathbf{1 0}$ & $\overline{\mathbf{1 0}}$ & $\mathbf{5}$ & $\overline{\mathbf{5}}$ \\
\hline spin 0 & $\left(1,0 ; \frac{1}{2}, \pm 1\right)$ & $\left(\frac{5}{8},-\frac{3}{2} ; \frac{1}{2}, \pm 1\right)$ & $\left(\frac{5}{8}, \frac{3}{2} ; \frac{1}{2}, \pm 1\right)$ & $\left(\frac{5}{8}, \frac{1}{2} ; \frac{1}{2}, \pm 1\right)$ & $\left(\frac{5}{8},-\frac{1}{2} ; \frac{1}{2}, \pm 1\right)$ \\
\hline spin 1 & $(1,0 ; 0,0)$ & $\left(\frac{5}{8},-\frac{3}{2} ; 0,0\right)$ & $\left(\frac{5}{8}, \frac{3}{2} ; 0,0\right)$ & $\left(\frac{5}{8}, \frac{1}{2} ; 0,0\right)$ & $\left(\frac{5}{8},-\frac{1}{2} ; 0,0\right)$ \\
\hline
\end{tabular}

Table 6.2 Massless spectrum for $S U(5)$

In this case further gluons can extend the gauge symmetry to $S O(10), E_{6}$ and also to $S U(6)$. Like in all string models where only Kac-Moody algebras at level one occur, one has to use a mechanism for breaking the GUT down to the standard model different from the spontaneous symmetry breaking by attaching a nonzero vacuum expectation value to some Higgs fields in the adjoint representation. For instance, if the fundamental group of the CYM is nontrivial one has the possibility to use Wilson lines.

For the gauge group $E_{3}$ the massless spectrum has the internal $(c, \bar{c})=(12,9)$ quantum numbers listed in Table 6.3.

\begin{tabular}{|l|l|l|l|l|l|l|}
\hline & $\mathbf{0}=(0,0)$ & $\mathbf{2}=(2,0)$ & $\mathbf{3}=(3,0)$ & $\overline{\mathbf{3}}=(\overline{3}, 0)$ & $\mathbf{6}=(3,2)$ & $\overline{\mathbf{6}}=(\overline{3}, 2)$ \\
\hline spin 0 & $\left(1,0 ; \frac{1}{2}, \pm 1\right)$ & $\left(\frac{3}{4}, 0 ; \frac{1}{2}, \pm 1\right)$ & $\left(\frac{3}{4}, 1 ; \frac{1}{2}, \pm 1\right)$ & $\left(\frac{3}{4},-1 ; \frac{1}{2}, \pm 1\right)$ & $\left(\frac{3}{4},-2 ; \frac{1}{2}, \pm 1\right)$ & $\left(\frac{3}{4}, 2 ; \frac{1}{2}, \pm 1\right)$ \\
\hline spin 1 & $(1,0 ; 0,0)$ & $\left(\frac{3}{4}, 0 ; 0,0\right)$ & $\left(\frac{3}{4}, 1 ; 0,0\right)$ & $\left(\frac{3}{4},-1 ; 0,0\right)$ & $\left(\frac{3}{4},-2 ; 0,0\right)$ & $\left(\frac{3}{4}, 2 ; 0,0\right)$ \\
\hline
\end{tabular}

Table 6.3 Massless spectrum for $S U(3) \times S U(2)$

After this partially quite technical presentation of exactly solvable $(0,2)$ CFTs, we calculate the massless spectra for some exemplary models in the following section.

\section{Examples}

Since the most frequently discussed example of a CYM is the quintic hypersurface in $\mathbb{C P}^{4}$, we will also focus our attention on the corresponding $N=2$ CFT of five copies of the $k=3$ unitary model, denoted as $(3)^{5}$. As some first results of a computer calculation we present how appropriate choices of simple currents yield $(0,2)$ models with gauge groups $E_{6}, S O(10), S U(5), S U(3) \times S U(2)$ and even $E_{7}, S U(7), S U(6), S O(12), S U(6) \times S U(2)$ and $S U(4) \times S U(2)$. Analogously to the exactly solvable $(2,2)$ string vacua these generic gauge groups are extended further by a factor $G$ which is a product of groups of small 
rank, usually $U(1)$ factors. In most cases these extensions are not written down explicitly in the following.

\section{- Gauge group $S O(10)$}

We start with a model like in Table 3.1 where we choose $(3)^{5}$ as the internal $c=9$ part. Besides all the projections in (3.21) we only include one further simple current (for simplicity we will not write down the ' $\otimes$ ' in the following)

$$
\Upsilon=\Phi_{0,-1}^{3} \otimes\left(\Phi_{0,0}^{0}\right)^{4} \otimes \Phi_{1,2}^{U(1)_{2}} \otimes \Phi_{0}^{S O(8)}
$$

of dimension $(h, q)=(1,0)$. Note, that (7.1) contains both factors from the $N S$ and the $R$ sector. The resulting massless spectrum is given in Table 7.1.

\begin{tabular}{|l|l|l|l|l|}
\hline & $\mathbf{0}$ & $\mathbf{1 0}$ & $\mathbf{1 6}$ & $\overline{\mathbf{1 6}}$ \\
\hline spin 0 & 350 & 74 & 80 & 0 \\
\hline spin 1 & 7 & 0 & 0 & 0 \\
\hline
\end{tabular}

Table 7.1 SO(10) model

Since no further gluons appear, the gauge group is $S O(10)$ by construction. Looking in more detail where the 80 generations are coming from, one realizes that 60 of them are ordinary $N=2$ states from the r.h.s. surviving all projections. However, the remaining 20 states arise in some orbits of $\Upsilon$ and contain both a nontrivial contribution of the $U(1)_{2}$ part and a mixing of $N S$ and $R$ states.

The numbers of particles in 10, 16 and $\overline{\mathbf{1 6}}$ agree with a model discussed in [8] which was defined on a complete intersection in the weighted projective space $W P_{(1,1,1,1,2,2)}^{5}$. Therefore our model could lie in the moduli space of that model which is based on a totally different approach. That would imply that our model, although built up on the $(3)^{5}$ tensor product, does not live on the quintic CYM.

Like in ordinary Gepner models there exists an isomorphic CFT, for which generations and antigenerations are interchanged. This mirror model can be obtained from the original one simply by including the following simple currents in front of the right moving states $\vec{\chi}(\bar{\tau})$ in $(3.21)$ :

$$
\begin{aligned}
J_{1}^{M} & =\Phi_{3,2}^{3} \Phi_{0,0}^{0} \ldots \Phi_{0,0}^{0} \Phi_{0,2}^{U(1)_{2}} \Phi_{0}^{S O(8)} \\
J_{2}^{M} & =\Phi_{0,0}^{0} \Phi_{3,2}^{3} \ldots \Phi_{0,0}^{0} \Phi_{0,2}^{U(1)_{2}} \Phi_{0}^{S O(8)} \\
& \vdots \\
J_{5}^{M} & =\Phi_{0,0}^{0} \Phi_{0,0}^{0} \ldots \Phi_{3,2}^{3} \Phi_{0,2}^{U(1)_{2}} \Phi_{0}^{S O(8)} \\
J_{6}^{M} & =\Phi_{0,0}^{0} \Phi_{0,0}^{0} \ldots \Phi_{0,0}^{0} \Phi_{2,2}^{U(1)_{2}} \Phi_{0}^{S O(8)} \\
J_{7}^{M} & =\Phi_{0,0}^{0} \Phi_{0,0}^{0} \ldots \Phi_{0,0}^{0} \Phi_{0,2}^{U(1)_{2}} \Phi_{v}^{S O(8)} .
\end{aligned}
$$

The generalization to the general case is straightforward. Thus, the class of models investigated in this paper exhibits mirror symmetry. For the class of linear $\sigma$ models in [7] mirror symmetry has not been established so far. 
It is easy to calculate at least net numbers of generations for the Distler/Kachru models. Due to $[7,17]$ the defining data of stable, holomorphic vector bundles $V=V_{1}, V_{2}$ are given by the exact sequence

$$
0 \rightarrow V \rightarrow \bigoplus_{a=1}^{r+M} \mathcal{O}\left(n_{a}\right) \rightarrow \bigoplus_{i=1}^{M} \mathcal{O}\left(m_{i}\right) \rightarrow 0
$$

Here $r=3,4,5,6$ yields gauge group $E_{9-r}$, and the $n_{a}$ and $m_{i}$ are positive integers chosen such that $c_{1}\left(V_{1}\right)=c_{1}\left(V_{2}\right)=0$ and the gauge anomaly vanishes, i.e. $c_{2}\left(V_{1}\right)+c_{2}\left(V_{2}\right)=c_{2}(T)$ with $T$ denoting the tangent bundle. The net numbers of generations are given by an index theorem

$$
N_{g e n}=\frac{1}{2}\left|\int_{M} c_{3}\left(V_{1}\right)\right|
$$

with

$$
c_{3}\left(V_{1}\right)=-\frac{1}{3}\left(\sum_{i} m_{i}^{3}-\sum_{a} n_{a}^{3}\right) J^{3} .
$$

By stochastically calculating some of these numbers for the quintic one realizes that only multiples of 5 occur and 80 really appears. Of course, much more work has to be done to definitely identify this model with a concrete $\sigma$ model. There are at least two candidates, for the model could be based on the quintic CYM or on the weighted projective space mentioned above.

\section{- Gauge group $E_{6}$}

Even though the construction yields gauge group $S O(10)$ it may happen that further extended gauge groups occur. For instance, without any further simple current $\Upsilon$ one gets the ordinary $(2,2)$ Gepner model with gauge group $E_{6}$. However, including the simple current

$$
\Upsilon=\Phi_{-3,0}^{3} \Phi_{1,1}^{0}\left(\Phi_{0,0}^{0}\right)^{3} \Phi_{1,2}^{U(1)_{2}} \Phi_{s}^{S O(8)}
$$

destroys the left moving $N=2$ supersymmetry but the $E_{6}$ remains unbroken. This can be read off explicitly from the $S O(10)$ massless spectrum listed in Table 7.2.

\begin{tabular}{|l|l|l|l|l|}
\hline & $\mathbf{0}$ & $\mathbf{1 0}$ & $\mathbf{1 6}$ & $\overline{\mathbf{1 6}}$ \\
\hline \hline spin 0 & 432 & 102 & 101 & 1 \\
\hline spin 1 & 5 & 0 & 1 & 1 \\
\hline
\end{tabular}

Table 7.2 Gepner-like model

Thus, this model lies in the enhanced $(0,2)$ moduli space of the quintic and is presumably connected to the $(2,2)$ moduli space by marginal deformations with $E_{6}$ singlets on the left moving side. Therefore, it is very suggestive of the $S O(10)$ deformation of the quintic. But one does not need to reproduce the Gepner spectrum. Dividing out the simple current

$$
\Upsilon=\Phi_{-3,0}^{3} \Phi_{1,1}^{0}\left(\Phi_{0,0}^{0}\right)^{3} \Phi_{1,2}^{U(1)_{2}} \Phi_{c}^{S O(8)}
$$


which is only slightly different from the current (7.6) one obtains a totally different spectrum as given in Table 7.3.

\begin{tabular}{|l|l|l|l|l|}
\hline & $\mathbf{0}$ & $\mathbf{1 0}$ & $\mathbf{1 6}$ & $\mathbf{1 6}$ \\
\hline \hline spin 0 & 368 & 62 & 41 & 21 \\
\hline spin 1 & 5 & 0 & 1 & 1 \\
\hline
\end{tabular}

Table $7.3 \quad E_{6}$ model

$N_{g e n}=20$ also appears in the list of Distler/Kachru spectra.

- Gauge group $S O(12)$

It is known that the Gepner model $(1)^{3}(2)(6)^{2}$ describes a compactification on $K_{3} \times T^{2}$ and yields both an extension of the gauge group to $E_{7}$ and an enhanced $N=2$ space-time supersymmetry. In [12] it has been argued that such models with at least $(0,4)$ world sheet supersymmetry have dual realizations as type II compactifications on CYMs. Using the simple current

$$
\Upsilon=\Phi_{0,-1}^{1}\left(\Phi_{0,0}^{0}\right)^{2} \Phi_{0,0}^{2}\left(\Phi_{0,0}^{0}\right)^{2} \Phi_{1,2}^{U(1)_{2}} \Phi_{0}^{S O(8)}
$$

in the $(1)^{3}(2)(6)^{2}$ model one obtains the spectrum in Table 7.4 .

\begin{tabular}{|l|l|l|l|l|}
\hline & $\mathbf{0}$ & $\mathbf{1 0}$ & $\mathbf{1 6}$ & $\overline{\mathbf{1 6}}$ \\
\hline spin 0 & 220 & 34 & 12 & 12 \\
\hline spin 1 & 14 & 2 & 0 & 0 \\
\hline
\end{tabular}

Table 7.4 $\quad N=2$ and $S O(12)$ model

This model has still $N=2$ space-time supersymmetry which requires $(0,4)$ world sheet supersymmetry. In addition, the two vectors in $\mathbf{1 0}$ extend the gauge group to $S O(12)$. Thus, there are three hypermultiplets in the two spinor representations $\mathbf{3 2}$ and $\overline{\mathbf{3 2}}$ of $S O(12)$. Nevertheless, the 14 singlets may even form a further extension by a nonabelian factor $G$. In principle, starting with a general CYM different from $K_{3} \times T^{2}$ it can also happen that $(0,4)$ models occur. Now, let us come to gauge groups of smaller rank.

\section{- Gauge group $S U(6)$}

Starting with a model like in Table 4.1 the gauge group is at least $S U(5)$. Analogously to the former case there occur extensions to larger gauge groups, in particular to $S O(10)$ and $E_{6}$. However, it can also happen that the gauge group is $S U(6)$. Including the simple current

$$
\Upsilon=\left(\Phi_{0,-1}^{3}\right)^{2}\left(\Phi_{0,0}^{0}\right)^{3}\left(\Phi_{1,2}^{U(1)_{2}}\right)^{2} \Phi_{0}^{S O(6)}
$$

in (4.13) one obtains the massless $S U(5)$ spectrum shown in Table 7.5. 


\begin{tabular}{|l|l|l|l|l|l|}
\hline & $\mathbf{0}$ & $\mathbf{1 0}$ & $\overline{\mathbf{1 0}}$ & $\mathbf{5}$ & $\overline{\mathbf{5}}$ \\
\hline \hline spin 0 & 348 & 54 & 4 & 69 & 119 \\
\hline spin 1 & 8 & 0 & 0 & 1 & 1 \\
\hline
\end{tabular}

Table 7.5 $S U(6)$ model

Thus, there are further gluons in the $\mathbf{5}$ and $\overline{\mathbf{5}}$ representation of $S U(5)$ which on account of

$$
35=24+5+\overline{5}+1
$$

leads to the enhanced gauge group $S U(6)$.

\section{- Gauge group $S U(5)$}

A model with $S U(5)$ gauge group can be achieved by choosing the following two simple currents:

$$
\begin{aligned}
& \Upsilon_{1}=\Phi_{0,-1}^{3}\left(\Phi_{0,0}^{0}\right)^{4} \Phi_{1,2}^{U(1)_{2}} \Phi_{0,2}^{U(1)_{2}} \Phi_{0}^{S O(6)} \\
& \Upsilon_{2}=\left(\Phi_{0,-1}^{3}\right)^{2}\left(\Phi_{0,0}^{0}\right)^{3}\left(\Phi_{1,2}^{U(1)_{2}}\right)^{2} \Phi_{0}^{S O(6)} .
\end{aligned}
$$

The massless spectrum is listed in Table 7.6.

\begin{tabular}{|l|l|l|l|l|l|}
\hline & $\mathbf{0}$ & $\mathbf{1 0}$ & $\overline{\mathbf{1 0}}$ & $\mathbf{5}$ & $\overline{\mathbf{5}}$ \\
\hline \hline spin 0 & 338 & 64 & 0 & 55 & 119 \\
\hline spin 1 & 10 & 0 & 0 & 0 & 0 \\
\hline
\end{tabular}

Table 7.6 $\quad S U(5)$ model

The gauge anomaly cancellation condition for $S U(5)$ requires the same number of chiral fermions in the $\mathbf{1 0}$ and $\overline{\mathbf{5}}$ representation. This condition is satisfied in our example yielding a net number of $N_{\text {gen }}=64$ generations which is not divisible by 5 . However, there exists a model with $N_{\text {gen }}=320$, so that we expect our model to be an orbifold of that model. It is a general problem of our construction that we do not have control over the geometric interpretation of dividing out a certain set of simple currents. It can correspond either to an orbifold construction or to a new vector bundle.

There is another example which shows that the number of generations in $\overline{\mathbf{1 0}}$ does not need to be zero. Therefore, we consider the tensor product of minimal models (1)(4) ${ }^{4}$ also adding up to $c=9$. This model has already turned out to yield a lot of different massless spectra [15]. For instance, choosing the two simple currents

$$
\begin{aligned}
& \Upsilon_{1}=\Phi_{0,-1}^{1}\left(\Phi_{0,0}^{0}\right)^{4} \Phi_{-1,2}^{U(1)_{2}} \Phi_{0,2}^{U(1)_{2}} \Phi_{v}^{S O(6)} \\
& \Upsilon_{2}=\Phi_{0,0}^{0} \Phi_{3,-1}^{4}\left(\Phi_{0,0}^{0}\right)^{3}\left(\Phi_{1,2}^{U(1)_{2}}\right)^{2} \Phi_{0}^{S O(6)}
\end{aligned}
$$

one obtains the spectrum presented in Table 7.7. 


\begin{tabular}{|l|l|l|l|l|l|}
\hline & $\mathbf{0}$ & $\mathbf{1 0}$ & $\overline{\mathbf{1 0}}$ & $\mathbf{5}$ & $\overline{\mathbf{5}}$ \\
\hline \hline spin 0 & 386 & 51 & 3 & 66 & 114 \\
\hline spin 1 & 10 & 0 & 0 & 0 & 0 \\
\hline
\end{tabular}

Table 7.7 Nonzero generation number in $\overline{\mathbf{1 0}}$

A stochastic search for net numbers of generations of the corresponding Distler/Kachru model $(1)(4)^{4}$ yields only numbers divisible by 3 . In particular, 48 net generations occur. It is clear, that the smaller the rank of the generic gauge group the larger the set of possible extensions of the gauge group. For $S U(3) \times S U(2)$ models we find also extensions to the semisimple groups $S U(6) \times S U(2)$ and $S U(4) \times S U(2)$.

- Gauge group $S U(6) \times S U(2)$

For the quintic $(3)^{5}$ the simple current

$$
\Upsilon=\left(\Phi_{0,-1}^{3}\right)^{3}\left(\Phi_{0,0}^{0}\right)^{2}\left(\Phi_{1,2}^{U(1)_{2}}\right)^{3} \Phi_{0}^{S O(4)}
$$

yields the $E_{3}$ spectrum in Table 7.8 .

\begin{tabular}{|l|l|l|l|l|l|l|}
\hline & $\mathbf{0}$ & $\mathbf{2}$ & $\mathbf{3}$ & $\overline{\mathbf{3}}$ & $\mathbf{6}$ & $\overline{\mathbf{6}}$ \\
\hline \hline spin 0 & 442 & 170 & 81 & 181 & 50 & 0 \\
\hline spin 1 & 13 & 0 & 3 & 3 & 0 & 0 \\
\hline
\end{tabular}

Table $7.8 \quad S U(6) \times S U(2)$ model

As expected by gauge anomaly cancellation for the $S U(3)$ factor the number $(\#(\mathbf{3})-\#(\overline{\mathbf{3}}))$ is twice the number $(\#(\overline{\mathbf{6}})-\#(\mathbf{6}))$.

- Gauge group $S U(4) \times S U(2)$

Analogously to the $S U(5)$ case one can obtain smaller gauge groups by including more simple currents. For instance,

$$
\begin{aligned}
& \Upsilon_{1}=\left(\Phi_{0,-1}^{3}\right)^{2}\left(\Phi_{0,0}^{0}\right)^{3}\left(\Phi_{1,2}^{U(1)_{2}}\right)^{2} \Phi_{0,2}^{U(1)_{2}} \Phi_{0}^{S O(4)} \\
& \Upsilon_{2}=\left(\Phi_{0,-1}^{3}\right)^{3}\left(\Phi_{0,0}^{0}\right)^{2}\left(\Phi_{1,2}^{U(1)_{2}}\right)^{3} \Phi_{0}^{S O(4)}
\end{aligned}
$$

gives the spectrum in Table 7.9.

\begin{tabular}{|l|l|l|l|l|l|l|}
\hline & $\mathbf{0}$ & $\mathbf{2}$ & $\mathbf{3}$ & $\overline{\mathbf{3}}$ & $\mathbf{6}$ & $\overline{\mathbf{6}}$ \\
\hline \hline spin 0 & 386 & 140 & 68 & 148 & 42 & 2 \\
\hline spin 1 & 11 & 0 & 1 & 1 & 0 & 0 \\
\hline
\end{tabular}

Table $7.9 \quad S U(4) \times S U(2)$ model 
In order to reduce the gauge group to $E_{3}$ one has to include three simple currents.

- Gauge group $S U(3) \times S U(2) \times G$

Choosing

$$
\begin{aligned}
& \Upsilon_{1}=\Phi_{0,-1}^{3}\left(\Phi_{0,0}^{0}\right)^{4} \Phi_{1,2}^{U(1)_{2}}\left(\Phi_{0,2}^{U(1)_{2}}\right)^{2} \Phi_{0}^{S O(4)} \\
& \Upsilon_{2}=\left(\Phi_{0,-1}^{3}\right)^{2}\left(\Phi_{0,0}^{0}\right)^{3}\left(\Phi_{1,2}^{U(1)_{2}}\right)^{2} \Phi_{0,2}^{U(1)_{2}} \Phi_{0}^{S O(4)} \\
& \Upsilon_{2}=\left(\Phi_{0,-1}^{3}\right)^{3}\left(\Phi_{0,0}^{0}\right)^{2}\left(\Phi_{1,2}^{U(1)_{2}}\right)^{3} \Phi_{0}^{S O(4)}
\end{aligned}
$$

gives the model with 50 net generations listed in Table 7.10.

\begin{tabular}{|l|l|l|l|l|l|l|}
\hline & $\mathbf{0}$ & $\mathbf{2}$ & $\mathbf{3}$ & $\overline{\mathbf{3}}$ & $\mathbf{6}$ & $\overline{\mathbf{6}}$ \\
\hline spin 0 & 370 & 134 & 54 & 154 & 50 & 0 \\
\hline spin 1 & 13 & 0 & 0 & 0 & 0 & 0 \\
\hline
\end{tabular}

Table $7.10 \quad S U(3) \times S U(2)$ model

Let us compute the additional factor $G$ for phenomenological reasons. Since 6 of the 13 singlet vector fields originate from the simple currents $\Upsilon_{i}$, they do not commute with the remaining $7 U(1)$ factors. A detailed analysis shows that the gauge group contains a further $S U(3)$ factor. Thus, the entire gauge group of this model is $S U(3) \times S U(2) \times$ $(U(1))^{5} \times S U(3)$. Besides the leptonic partners of the 50 quarks there occur 84 further Higgses in the $(0,2)$ representation of $E_{3}$.

What can also happen is the occurrence of gauge groups of rank higher than six.

\section{- Gauge group $E_{7}$}

For this extended group we again start with the model $(1)(4)^{4}$ and select only the simple current

$$
\Upsilon_{1}=\Phi_{0,-1}^{1}\left(\Phi_{0,0}^{0}\right)^{4} \Phi_{1,2}^{U(1)_{2}} \Phi_{s}^{S O(8)}
$$

Due to the decomposition of the adjoint representation of $E_{7}$ into irreducible representations of $S O(10)$

$$
133=45+10+10+16+16+\overline{\mathbf{1 6}}+\overline{\mathbf{1 6}}+1+1+1+1
$$

the massless spectrum in Table 7.11 yields 36 generations in the $\mathbf{5 6}$ representation of $E_{7}$.

\begin{tabular}{|l|l|l|l|l|}
\hline & $\mathbf{0}$ & $\mathbf{1 0}$ & $\mathbf{1 6}$ & $\overline{\mathbf{1 6}}$ \\
\hline spin 0 & 416 & 72 & 36 & 36 \\
\hline spin 1 & 7 & 2 & 2 & 2 \\
\hline
\end{tabular}

Table $7.11 \quad E_{7}$ model 
$N_{g e n}=36$ can also be reproduced using (7.4). Furthermore, one can even produce the gauge group $S U(7)$.

- Gauge group $S U(7)$

Again we choose (1)(4) ${ }^{4}$ and include the following two simple currents:

$$
\begin{aligned}
& \Upsilon_{1}=\Phi_{0,-1}^{1}\left(\Phi_{0,0}^{0}\right)^{4} \Phi_{1,2}^{U(1)_{2}} \Phi_{0,2}^{U(1)_{2}} \Phi_{s}^{S O(6)} \\
& \Upsilon_{2}=\Phi_{0,0}^{0} \Phi_{3,-1}^{4}\left(\Phi_{0,0}^{0}\right)^{3}\left(\Phi_{1,2}^{U(1)_{2}}\right)^{2} \Phi_{0}^{S O(6)} .
\end{aligned}
$$

The extension of $S U(5)$ to $S U(7)$ can be seen from Table 7.12 taking into account the decomposition

$$
49=24+5+5+\overline{5}+\overline{5}+1+1+1+1
$$

\begin{tabular}{|l|l|l|l|l|l|}
\hline & $\mathbf{0}$ & $\mathbf{1 0}$ & $\overline{\mathbf{1 0}}$ & $\mathbf{5}$ & $\overline{\mathbf{5}}$ \\
\hline \hline spin 0 & 463 & 49 & 7 & 90 & 132 \\
\hline spin 1 & 15 & 0 & 0 & 2 & 2 \\
\hline
\end{tabular}

Table $7.12 \quad S U(7)$ model

A net number of 42 generations also occur for the Distler/Kachru models. Finally, we present an example with four net generations and gauge group $S O(10)$. This spectrum results from the quintic $(3)^{5}$ and the simple currents

$$
\begin{aligned}
& \Upsilon_{1}=\Phi_{-2,-1}^{3}\left(\Phi_{-1,2}^{3}\right)^{2} \Phi_{-3,0}^{3} \Phi_{0,0}^{0} \Phi_{1,2}^{U(1)_{2}} \Phi_{c}^{S O(8)} \\
& \Upsilon_{2}=\Phi_{0,0}^{0} \Phi_{-3,0}^{3}\left(\Phi_{0,0}^{0}\right)^{2} \Phi_{1,1}^{0} \Phi_{1,2}^{U(1)_{2}} \Phi_{v}^{S O(8)}
\end{aligned}
$$

with the spectrum listed in Table 7.13. This model is likely an orbifold of a Distler/Kachru model.

\begin{tabular}{|l|l|l|l|l|}
\hline & $\mathbf{0}$ & $\mathbf{1 0}$ & $\mathbf{1 6}$ & $\mathbf{\overline { \mathbf { 1 6 } }}$ \\
\hline \hline spin 0 & 254 & 32 & 18 & 14 \\
\hline spin 1 & 7 & 0 & 0 & 0 \\
\hline
\end{tabular}

Table 7.13 Four net generations

All these examples show that one can really find suitable simple currents $\Upsilon$ breaking both the left moving $N=2$ supersymmetry and the gauge group $E_{6}$. Furthermore, the massless spectra obtained can all be found in the context of $(0,2)$ (non)linear $\sigma$ models encouraging further investigations about their relationship. 


\section{Conclusion}

In this paper we have presented a method to construct modular invariant partition functions of four space-time dimensional heterotic string vacua with $(0,2)$ world sheet supersymmetry and generic gauge groups $E_{r}$ with $3 \leq r \leq 6$. This constructively proves the existence of bona fide CFTs with all the properties known for the conformal fixed points of $(0,2)$ supersymmetric CYM/LG models. In particular, these vacua are not suffering from destabilizing instanton corrections. Clearly, our construction is not unique and there might exist different construction schemes of $(0,2)$ CFTs, especially those starting with a truly heterotic modular invariant partition function. However, $(2,2)$ models have taught us that due to the GSO projection the spectra obtained are highly degenerate. Thus, we hope that more than only a very small subset of all exactly solvable $(0,2)$ vacua can be realized by the simple current method.

Since both orbifolds of the CYM and the choice of a more general stable vector bundle for the left moving $\sigma$ model fermions are encoded in the same manner in this class of CFTs, a direct correspondence between simple currents and the defining data of the latter bundles might be hardly to reveal. One ansatz to construct such a map could be the coincidence of the model in Table 7.1 and a complete intersection in a weighted projective space. If such a one to one identification could be achieved for at least a few models, it would be possible to calculate and compare further properties of physical importance. For instance, one could address questions like the exactness of a first order calculation of some of the Yukawa couplings in the CYM/LG framework. Furthermore, since the class of exactly solvable models exhibits exact mirror symmetry, one expects such an isomorphism for the CYM/LG formulation, as well.

Thus, in order to learn more about the degeneracy of the spectra and the appearing net numbers of generations one has to extend further the set of explicitly known models [4].

\section{Acknowledgements}

It is a pleasure to thank L. Dolan and W. Nahm for discussion, S. Kachru for his hint on the coincidence concerning the model in Table 7.1, S. Chaudhuri for interesting questions on extensions of the gauge groups and in particular R. Schimmrigk for pointing our attention to string models with $(0,2)$ world sheet supersymmetry. This work is supported by U.S. DOE grant No. DE-FG05-85ER-40219.

\section{References}

[1] I. Antoniadis, C. Bachas and C. Kounnas, Nucl. Phys. B289 (1987) 87, L. Ibáñez, H. Nilles and F. Quevedo, Phys. Lett. B187 (1987) 25, H. Kawai, D. Lewellen and S. Tye, Nucl. Phys. B288 (1987) 1, W. Lerche, D. Lüst and A.N. Schellekens, Nucl. Phys. B287 (1987) 477, K. Narain, M. Sarmadi and C. Vafa, Nucl. Phys. B288 (1987) 551

[2] T. Banks, L.J. Dixon, D. Friedan and E. Martinec, Phenomenology and conformal field theory, or Can string theory predict the weak mixing angle?, Nucl. Phys. B299 (1988) 613 
[3] R. Blumenhagen and A. Wißkirchen, Generalized string functions of $N=1$ spacetime supersymmetric string vacua, Phys. Lett. B349 (1995) 63

[4] R. Blumenhagen and A. Wißkirchen, work in progress

[5] P. Candelas, G.T. Horowitz, A. Strominger and E. Witten, Vacuum configurations for superstrings, Nucl. Phys. B258 (1985) 46

[6] M. Dine, N. Seiberg, X.G. Wen and E. Witten, Nonperturbative effects on the string world sheet I+II, Nucl. Phys. B278 (1986) 769, Nucl. Phys. B289 (1987) 319

[7] J. Distler and B. Greene, Aspects of $(2,0)$ string compactifications, Nucl. Phys. B304 (1988) 1

[8] J. Distler and S. Kachru, (0,2) Landau-Ginzburg theory, Nucl. Phys. B413 (1994) 213

[9] J. Distler and S. Kachru, Singlet couplings and (0,2) models, Nucl. Phys. B430 (1994) 13

[10] T. Eguchi, H. Ooguri, A. Taormina and S.K. Yang, Superconformal algebras and string compactification on manifolds with $S U(n)$ holonomy, Nucl. Phys. B315 (1989) 193

[11] D. Gepner, Space-time supersymmetry in compactified string theory and superconformal models, Nucl. Phys. B296 (1988) 757

[12] S. Kachru and C. Vafa, Exact results for $N=2$ compactifications of heterotic strings, preprint HUTP-95/A016 (hep-th/9505105)

[13] S. Odake, Character formulas of an extended superconformal algebra relevant to string compactification, Int. J. Mod. Phys. A5 (1990) 897

[14] A.N. Schellekens and N.P. Warner, Anomalies, characters and strings, Nucl. Phys. B287 (1987) 317

[15] A.N. Schellekens and S. Yankielowicz, Extended chiral algebras and modular invariant partition functions, Nucl. Phys. B327 (1989) 673

[16] A.N. Schellekens and S. Yankielowicz, Modular invariants from simple currents. An explicit proof, Phys. Lett. B227 (1989) 387

[17] A.N. Schellekens and S. Yankielowicz, New modular invariants for $N=2$ tensor products and four-dimensional strings, Nucl. Phys. B330 (1990) 103

[18] E. Silverstein and E. Witten, Criteria for conformal invariance of $(0,2)$ models, Nucl. Phys. B444 (1995) 161

[19] E. Witten, New issues in manifolds of SU(3) holonomy, Nucl. Phys. B268 (1986) 79

[20] E. Witten, Phases of $N=2$ theories in two dimensions, Nucl. Phys. B403 (1993) 159 